\section{GeneXpert TB 8: A point-of-care} diagnostic pillot

To the Editor: The point-of-care (POC) GeneXpert MTB/RIF detects Mycobacterium tuberculosis (MTB) and drug resistance in under 2 hours and was hailed a 'game-changer' for tuberculosis (TB) control. ${ }^{[1}$ Recent results from Evaluating a New Diagnostic Trial (EXTEND) show that while implementing the GeneXpert had more confirmations of TB than sputum microscopy, levels of loss to follow-up and mortality were not reduced in the GeneXpert group. ${ }^{[2]}$ These results are discouraging. However, GeneXpert was not used as a true POC service. Using the diagnostic at POC, where results are handed to patients at the time and place of sample collection, may yield different outcomes.

The Desmond Tutu HIV Foundation has piloted the GeneXpert in the Tutu Tester Mobile Clinic operating on the Cape Flats in Cape Town, Western Cape Province, South Africa.

The context. The Western Cape has one of the highest incidence rates of TB in the world. ${ }^{[3]}$ The uncontrolled spread of TB has led to its being declared a national emergency. ${ }^{[4]}$ Accessible healthcare promotes health-seeking and enables patients to become more active in their own healthcare. ${ }^{[5]}$ Limited access means that treatable diseases such as HIV and TB go undiagnosed, ${ }^{[6]}$ and many enter care with advanced disease. Providing POC testing can facilitate entry into the HIV/TB treatment cascade $^{[7]}$ for many in the Cape Town metropole. To facilitate treatment access further, the Foundation has piloted the GeneXpert in underresourced communities with a high burden of disease.

Intervention. Implementing the GeneXpert in the mobile clinic has shortened the diagnostic period. Patient samples can be analysed for TB DNA and the results can be handed back within 2 hours. ${ }^{[8]}$ Most clients leave with their test result and a referral letter to their neares clinic. This allows for fast-tracking for medication prescriptions. Through active case finding, mobile clinics reach people who often do not access healthcare and patients are therefore infectious for shorter periods, helping to combat TB transmission. In addition, people who could not wait 2 hours for TB results were followed up telephonically and linkage to care was facilitated with the relevant clinic.

Data to date. In a 3-month period, 66 tests were conducted with HIV-positive clients with low $\mathrm{CD}^{+}$counts and clients with World Health Organization TB symptoms. Of these, nine (13.6\%) tested positive for MTB. Rifampicin resistance was found in two $(22.2 \%)$ of nine TB-positive clients (3.0\% of 66 tested). Of the nine clients, seven were linked into care within 48 hours (one client linked into care 10 days after testing). All these clients received their first dose of medication on the day of linking to a healthcare facility.

Conclusion. These very preliminary data indicate that the GeneXpert can be used at the point of patient contact, and when doing so, therapy can be initiated on the same day or very soon thereafter.

Philip Smith, Anna van Esch, Melissa Wallace, Robin Wood,

\section{Linda-Gail Bekker}

Desmond Tutu HIV Centre, Institute for Infectious Disease and Molecular Medicine, Faculty of Health Sciences, University of Cape Town, South Africa

philip.smith@hiv-research.org.za

1. Evans CA. GeneXpert - a game-changer for tuberculosis control? PLoS Med 2011:8(7):e1001064 [http://dx.doi.org/10.1371/journal.pmed.1001064]

2. Fielding KL, McCarthy KM, Cox H, et al. Xpert as the first-line test in South Africa: Yield, intial los to follow up, proportion treated. Presented at the 2014 Conference on Retroviruses and Opportunistic Infections (CROI), Boston, MA, 3 - 6 March 2014

3. Claassens $\mathrm{M}$, van Schalkwyk C, den Haan L, et al. High prevalence of tuberculosis and insufficien case detection in two communities in the Western Cape, South Africa. PLoS ONE 2013:8(4):e58689. [http://dx.doi.org/10.1371/journal.pone.0058689]

4. Abdool Karim SS, Churchyard GJ, Abdool Karim Q, Lawn SD. HIV infection and tuberculosis in South Abdool Karim SS, Churchyard GJ, Abdool Karim Q, Lawn SD. HIV infection and tuberculosis in Sout/
Africa: An urgent need to escalate the public health response. Lancet 2009;374(9693):921-933. [http:// Africa: An urgent need to escalate the public
dx.doi.org/10.1016/S0140-6736(09)60916-8]

5. Govindasamy D, Kranzer K, van Schaik N, et al. Linkage to HIV, TB and non-communicable disease Govindasamy D, Kranzer K, van Schaik N, et al. Linkage to HIV, TB and non-communicable disease
care from a mobile testing unit in Cape Town, South Africa. PLoS ONE 2013;8(11):e80017. [http. $/$. dx.doi.org/10.1371/journal.pone.0080017]

6. MacPherson P, MacPherson EE, Mwale D, et al. Barriers and facilitators to linkage to ART in primary care: A qualitative study of patients and providers in Blantyre, Malawi. J Int AIDS Soc 2012;15:18020. [http://dx.doi.org/10.7448/IAS.15.2.18020]

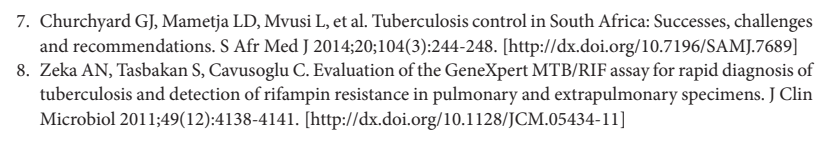

S Afr Med J 2014;104(8):524. DOI:10.7196/SAMJ.846 